\title{
Diversity and equitability ordering profiles applied to study forest structure
}

\author{
Rubén Valbuena ${ }^{\mathrm{a}, *}$, Petteri Packalén ${ }^{\mathrm{b}, \mathrm{c}}$, Susana Martín-Fernández ${ }^{\mathrm{d}}$, Matti Maltamo ${ }^{\mathrm{b}}$ \\ ${ }^{a}$ European Forest Institute HQ Torikatu 34, 80100 Joensuu, Finland \\ ${ }^{\mathrm{b}}$ University of Eastern Finland, Faculty of Forest Sciences, PO Box 111, Joensuu, Finland \\ ' Oregon State University, College of Forestry, 204 Peavy Hall, Corvallis, OR 97331-5704, USA \\ ${ }^{\mathrm{d}}$ Technical University of Madrid, School of Forestry, Ciudad Universitaria s/n., 28040 Madrid, Spain
}

\section{A R T I C L E I N F O}

\section{Article history:}

Received 13 December 2011

Received in revised form 29 March 2012

Accepted 30 March 2012

Available online 3 May 2012

\section{Keywords:}

Intrinsic diversity ordering

Intrinsic equitability ordering

Lorenz curves

Gini coefficient

Forest structure

Diversity indices

\begin{abstract}
A B S T R A C T
This article performs an in-depth examination on whether indices of diversity and equitability among tree size classes are adequate for studying the structural complexity of forests. Diversity profiles and the intrinsic diversity ordering of several field plots were compared. Results demonstrated that evensized stands are intrinsically non-comparable to uneven-sized stands with regard to their diversity of size classes. Indices describing the diversity of size classes are consequently inadequate, as they order forest structural types (FSTs) inconsistently. The concept of equitability, obtained when removing the richness component from entropy, seemed more adequate for this purpose. Indices of equitability among size classes provided more consistent measures, since the field plots had comparable intrinsic equitability ordering. Furthermore, ranking individual trees by their size is a better approach than ranking size classes, and therefore it is more correct to measure the inequality of tree sizes rather than equitability among size classes. A particular interpretation of Lorenz curves applies when they are used for the study of forest structures, as they should also be compared to a theoretical uniform distribution, and not just the diagonal line-of-absolute-equality. Advised indices are Gini coefficient (GC), De Camino homogeneity ( $\mathrm{CH}$ ), and structure index based on variance (STVI), as they all are consistent with the Lorenz ordering.
\end{abstract}

(ㄷ) 2012 Elsevier B.V. All rights reserved.

\section{Introduction}

One of the most relevant components of diversity in forests is the variation in tree size (McArthur and McArthur, 1961). The evaluation of ecological, economic, and other multi-functional aspects of forests can benefit from a good knowledge of size distributions. Natural dynamics lead to more complex forest structures in the absence of human intervention (Hett and Loucks, 1976), whereas certain management alternatives may lead to homogeneouslysized structures (Lähde et al., 1991). Seed regeneration and recruitment are affected by the complex effect of canopy structure in light penetration (Montgomery and Chazdon, 2001). Disturbances affect forest structure (Coomes et al., 2003), and therefore forest dynamics and development (Zenner, 2005).

Simple indices can be used for describing changes in the structural complexity of forests in a concise manner. They can be used as a basis for classifying forest stands into generalized forest structural types (FSTs). They can also be used as a surrogate of other important ecological properties, such as habitat quality for fauna. Stand structure may influence the organization of avian communities (James and Wamer, 1982), or the distribution of small

\footnotetext{
* Corresponding author. Tel.: +358 107734334 .

E-mail address: ruben.valbuena@efi.int (R. Valbuena).
}

mammals (Sullivan et al., 2001). Structure indices can be integrated in forest dynamics models to improve their predictive power (Lei et al., 2009). They can also be helpful in assessing the protective function of forest ecosystems (Bachofen and Zingg, 2001). Objective indicators of tree size diversity are needed in order to evaluate alternative management plans regarding their environmental benefits and sustainability (Buongiorno et al., 1994; Solomon and Gove, 1999). However, there is a lack of common criteria on describing the size structure of forests (Cienciala and Korhonen, 2011).

There have been several attempts to quantitatively summarize the structural complexity of forests by means of simple indices (Mc Elhinny et al., 2005). Pommerening (2002) provided an overview of all them and their general classification into non-spatial and spatial (neighborhood) indices, and also distinguishing between those measuring spatial autocorrelation, species mixture, or size classes diversity in both horizontal and vertical strata. Furthermore, structural complexity can also be analyzed as beta diversity, i.e., inter-stand heterogeneity, whereas alpha diversity measures are performed at a finer within-stand scale (cf., McRoberts et al., 2008). The focus of this article is only on alpha (plot-level) non-spatial indices of diameter classes diversity and equitability. The calculation of these indices follows four broad types of approaches in the research literature: (1) adaptations of species biodiversity indices based on the probability for 
inter-specific encounters (Shannon, Simpson, etc.) to the study of diversity of size classes; (2) adaptations of the equitability counterparts of those same diversity indices (Shannon or Simpson's evenness); (3) indices based on dispersion estimates of tree size (Gini, $\mathrm{CH}$, variance, STVI); and a last group of (4) methods based on descriptors of histogram's shape (skewness, Weibull parameters, etc.) which are not considered in this article.

\subsection{Diversity of tree size classes}

Biodiversity indices were originally intended to describe species richness and their relative abundance; the number of species, and the number of individuals per species, respectively (Magurran, 2004). When applying these indices to study the structural diversity of forest stands, richness is described by the number of diameter or height classes, whereas abundance is usually expressed in terms of their relative proportions of number of stems, basal area, volume or biomass (Pommerening, 2002). Some of these indices are more influenced by the range of size classes present (richness) and others also by the abundances (diversity), with some of the latter more influenced by the most abundant classes (dominance) and others focused on the relative differences among them (evenness or equitability). The most popular among all the alternatives is the Shannon's diversity index $\left(H^{\prime}\right)$. Although $H^{\prime}$ is more widely used as a measure of species mixture of forests (e.g., Motz et al., 2010; Neumann and Starlinger, 2001; Sterba and Ledermann, 2006), many authors have used this index for comparing tree sizes within a single species. One of the earliest approaches aimed at describing the vertical strata of forests by computing $H^{\prime}$ from proportions of foliage density at different heights within the canopy (James and Wamer, 1982; McArthur and McArthur, 1961). These studies have mainly been linked to bird ecology, as it has an intuitive causal relation with crown layer structure. However, tree diameters are easier to measure in the field and accordingly more commonly available in plot-based forest inventory than crown bulk and vertical strata. Moreover, diversity of diameter classes can be regarded as a good proxy for foliage height diversity (Buongiorno et al., 1994; Montgomery and Chazdon, 2001), and therefore it is more common to find studies computing $H^{\prime}$ from relative frequencies of stems among diameter classes (Gove et al., 1995). Solomon and Gove (1999) introduced the idea of computing the abundance vector from proportions of basal area instead of stem frequencies. Disadvantages of using diversity indices include the need for partitioning into bins (size classes), with the subsequent loss of information and dependency on the subjective choice of bin size (Staudhammer and LeMay, 2001), and also their sensitivity to sample size (Lexerød and Eid, 2006) and plot dimensions (Barbeito et al., 2009).

\subsection{Equitability among size classes}

The concept of equitability is closely associated to diversity (Wittebolle et al., 2009), as it is a means of assessing evenness among relative abundances. For a given number of size classes (richness), the forest having abundances equally distributed among them is also the one obtaining the maximum plausible value of diversity. Hence, diversity is a simultaneous measure of both richness and equitability (Hill, 1973). For this reason, most equitability (evenness) indices are based on some means of normalizing its diversity counterpart by the number of size classes, or by its maximum theoretical value. This approach was followed by Buongiorno et al. (1994), who found that structural diversity can be preserved without compromising the economic value of the forest. O'hara et al. (2007) observed that equitability is naturally maintained by forest dynamics in multi-aged forests. Lexerød and Eid (2006) compared diversity and equitability indices, finding inconsistencies with regards to their potential for discriminating among forest types and also to their ability to be arranged in a reliable order of structural complexity.

\subsection{Diversity ordering}

Many authors have reported unsatisfactory results when using diversity indices (Barbeito et al., 2009; Harper et al., 2003; Kint et al., 2000; O'hara et al., 2007; Rouvinen and Kuuluvainen, 2005), giving a general feeling that diversity indices are inconsistent with the properties that they are meant to describe. Others found forest structure to be more related to measures of dispersion or equitability (Lexerød and Eid, 2006; McRoberts et al., 2008) than to diversity indices. However, only weak statistical indications have been used to reach these conclusions, such as correlations between diverse indices or statistical differences found among FSTs. Little effort has been invested in examining the implications of adapting those indices to the study of tree size classes, whether it complies with the definition of diversity and its mathematical interpretation, and assuring that the basic assumptions underlying the calculation of these indices are not violated.

The problem of inconsistent ordering of communities or assemblages (here considered at plot-level) according to diversity indices is not new, and nor is it confined to ecology studies (Patil and Taillie, 1982). For this reason, many authors have stated that, prior to using any diversity index, a diversity ordering method should be tested in order to determine whether the communities considered are comparable or not (Lambshead et al., 1983). A useful outcome of these methods, a diversity profile, is a graphical representation of a collection of indices which belong to the same general form. If the diversity profiles of two communities intersect, the result of the indices related are inconsistent and therefore unreliable for comparing those two communities.

Liu et al. (2007) provided a useful categorization of diversity ordering methods, differentiating between those based upon: (1) information theory (entropy, numbers equivalent, diversity), (2) the statistical expectancy of the number of size classes (rarefaction), (3) ranking, or (4) the theory of intrinsic diversity ordering (right tail-sum, majorization, k-dominance). The first category of profiling methods represents a collection of indices which belong to the same parametric family. For instance, a so-called diversity profile is obtained when size classes count $(s), H^{\prime}$, and Simpson $\left(D_{S i}\right)$ are depicted together as entropy indices of order 0,1 and 2 respectively. The second category is based on the expectancy and variance for the total number of size classes (Hurlbert, 1971), which is a more strict estimate of richness than sample $s$ (see e.g., McRoberts et al., 2008). The third category contains a single index based on ranking, which was introduced by Patil and Taillie (1982) as a better descriptor of the internal composition of the community. The fourth category has been suggested as the most reliable approach for assuring that the abundance distributions of the communities compared can intrinsically be ordered by the definition of diversity used (Lambshead et al., 1983). This can be carried out by comparing their intrinsic diversity profiles, which are obtained by accumulating the relative dominances of their abundances. Recent research (Gattone and Battista, 2009) has attempted to enhance the use of profiles for describing ecological diversity.

Methods for testing the intrinsic ordering of assemblages include right tail-sum, k-dominance plot, and majorization (cf., Liu et al., 2007). Despite the demonstrated importance of comparing diversity profiles, their use in adaptations of diversity indices for forestry applications is sparse. Swindel et al. (1987) and Gove et al. (1992) used them for assessing the effect of management alternatives on forest biodiversity. Gove et al. (1995) and Solomon and Gove (1999) subsequently applied the right tail-sum of stem 
frequencies to the study of diversity of size classes. Apart from these studies, no reports on the intrinsic diversity ordering of data can be found accompanying the above-mentioned size classadapted $H^{\prime}$, in the forestry literature. Among those described by Liu et al. (2007), in this article methods for (1) diversity profiling and (4) intrinsic ordering were chosen. Diversity profiles demonstrate whether indices arrange communities consistently, whereas intrinsic ordering is used for testing if their intrinsic structure makes them comparable by the diversity definition used by those indices. Diversity numbers from generalized entropy were selected to depict diversity profiles (Hill, 1973), and the majorization method was used for intrinsic ordering (Solomon, 1979).

\subsection{Lorenz ordering}

The Lorenz curve is another concept related to size ordering for characterizing equitability (Studeny et al., 2011). Even though the Lorenz curve was originally applied to a different problem area (inequality of economic income), it is equivalent to an intrinsic size diversity ordering method when richness is constant (Lambshead et al., 1983), as it is also based on accumulated dominance. Using Lorenz ordering for comparing biomass differences among individuals in a plant community was first suggested by Weiner and Solbrig (1984), who also pointed out the usefulness within this context of estimating diameter dispersion by means of the Gini coefficient (GC). The GC equals to half the relative mean difference in size among all the trees, and it is usually conceptualized as the area comprised between the Lorenz curve and the diagonal line-ofabsolute-equality (e.g., Wittebolle et al., 2009). It has been used to study how natural forest growth dynamics affect the equality among tree sizes (Knox et al., 1989; Lei et al., 2009). GC has been reported as a forest structure indicator which performs better than $H^{\prime}$ and other indices (Lexerød and Eid, 2006). Consequently, GC has gained recent attention as an evaluator of forest management practice (Duduman, 2011; Klopcic and Boncina, 2011). However, it has been noted that the same values of GC can be obtained by very different Lorenz curves, and consequently a Lorenz asymmetry coefficient (S) was also developed (Damgaard and Weiner, 2000).

De Camino's (1976) homogeneity index $(\mathrm{CH})$ was also developed when applying Lorenz ordering to forestry, though its use has been more marginal than GC's. For example, Bachofen and Zingg (2001) studied how forest management operations led to changes in $\mathrm{CH}$, and compared them to other unmanaged areas. Another estimate of relative dispersion was also used by Staudhammer and LeMay (2001) in their structure index based on variance (STVI), though its relation with Lorenz ordering has not been addressed before. An advantage of GC, S, CH and STVI against the other indices considered in this study is that there is no need for grouping the sample into discrete size classes. Therefore it is possible to keep all the information available and reduce subjectivity. One drawback of applying Lorenz ordering to study forest canopy structure is that it only makes sense for variables which are cumulative at stand level, namely basal area, volume or biomass, but it has no straightforward use for tree heights.

\subsection{Objectives}

The present article aims at expanding the applicability of using diversity and equitability profiles in studies regarding the size structure of forests. By observing the profiles of different FSTs simultaneously, we clarify the reasons why differing diameter distributions may lead to similar values for some indices. We investigated their intrinsic ordering properties, discussing which FSTs are comparable and which are not. Lorenz curves are also presented, in order to illustrate that measurements based on equitability are more suited for studying forest structure than those intended to describe diversity. This article also considers the difference between computing Lorenz curves by size classes or tree-by-tree, describing the advantages of the latter approach. The interpretation of Lorenz curves applied to study forest structure is fully discussed. All these constitute the basis for discouraging the use of some indices and advising alternative ones.

\section{Methods}

\subsection{Plot mensuration and forest structural types (FST)}

The data for this study was collected in field plots measured in the monospecific Scots pine (Pinus sylvestris L.) forest of Valsain (Spain; approx. lat.: $41^{\circ} 5^{\prime} \mathrm{N}$; lon.: $4^{\circ} 8^{\prime} \mathrm{W}$; $1.4 \mathrm{~km}$ a.s.l.). The plot radius was $20 \mathrm{~m}$, as 0.13 ha was considered a sufficiently large plot size not to influence index computation (Barbeito et al., 2009). Tree basal areas $\left(g_{t}, \mathrm{~m}^{2}\right)$ were aggregated at plot level $\left(g, \mathrm{~m}^{2}\right)$, being $g=\sum_{t=1}^{n} g_{t}$, where $n$ is the total number trees in the plot. As it was mandatory for some of the indices involved, the dataset was separated into discrete $2-\mathrm{cm}$ diameter classes. Bin width selection is a matter of subjectivity, and a fine scale was chosen to diminish the loss of information when partitioning (e.g., Lexerød and Eid, 2006). We computed the number of stems $\left(n_{i}\right)$ and the basal area $\left(g_{i}\right)$ included within each tree size class $i=1, \ldots, s ; s$ being the number of size classes found in a particular field plot. Valsaín forest was selected because it contains a great variety of forest structures, as a consequence of a group shelterwood forest management based on long regeneration periods. Shade-intolerant natural regeneration is encouraged by opening the canopy in patches, leading to a large spatial variability in its structural complexity. Six reference plots have been selected to illustrate throughout this article the results obtained by the FSTs present (Fig. 1):

FST I: Even-sized. They are single-cohort situations, which can be approximated to normal distributions in both stem-frequency and basal area proportions. In order to signify in all figures the range of indicator values that even-sized stands may obtain and trends that can be observed along their stages of development, two sub-types are differentiated throughout this article: (i) FST Ia represents canopies mainly dominated by middle-aged poles (quadratic mean diameter $D g<35 \mathrm{~cm}$ ); and (ii) FST Ib signifies very mature forest stands $(D g>35 \mathrm{~cm})$. All the remaining FSTs represent uneven-sized scenarios.

FST II: Uniform. In group shelterwood forest management, this FST appears only incidentally depending on disturbance history, or as a boundary state if a plot is located close to the drip-line of a canopy gap and contains trees from both groups. The main idea is that stem frequencies are more or less uniformly distributed over a multiplicity of size classes, which means that the proportions of basal area slightly increase towards the bigger size classes, showing a negatively skewed distribution (cf., Lexerød and Eid, 2006). Due to high canopy closure, no saplings are usually present at FST II, so that if a forest plot shows a significant amount of trees $<10 \mathrm{~cm}$ a classification as reverse-J (FST IV) may be considered instead.

FST III: Bimodal. Two-cohort situations with a well-established regeneration layer, obtained when recruitment has been achieved (Zenner, 2005). The gap between the dominant and subdominant strata must be clear, and basal areas must be roughly balanced between them. FST III may be hard to identify from a stem frequency histogram, as only the distribution of basal areas may distinguish it from a FST IVa. They are difficult to generalize, as different functions may apply for each cohort (Coomes et al., 2003). 

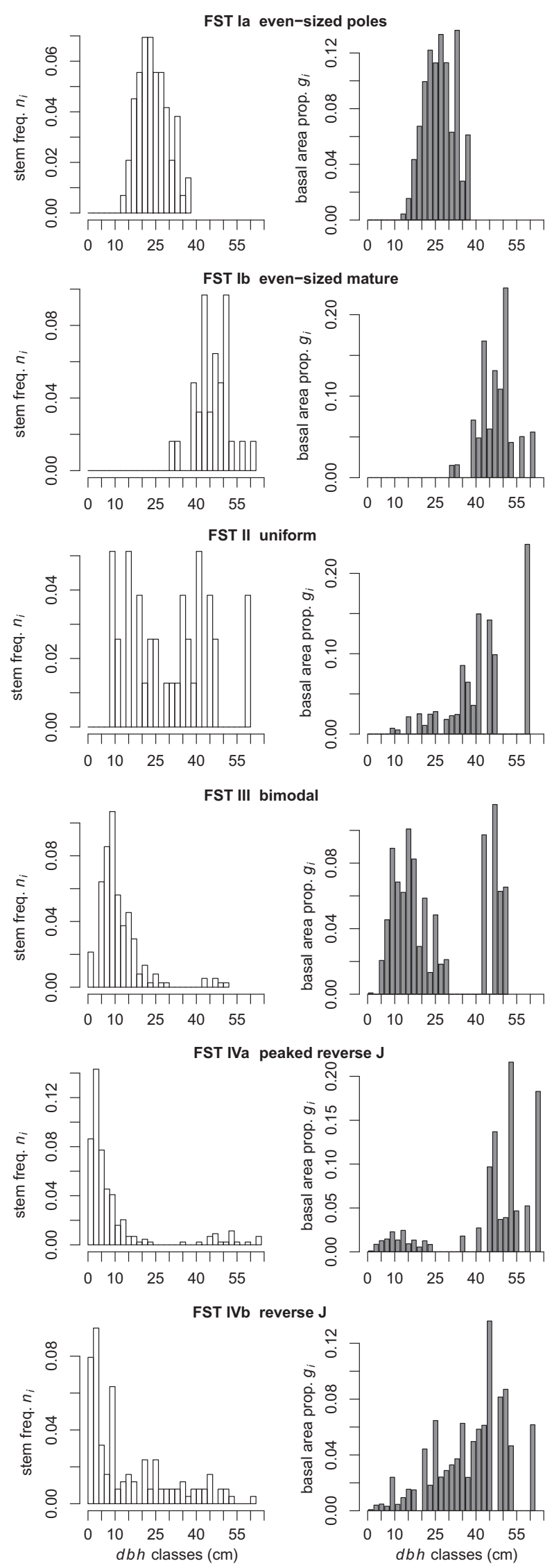

Fig. 1. Histograms of diameter distributions (white bars on the left) and proportions of basal area (dark bars on the right) per $2-\mathrm{cm}$ size classes for each forest structural type (FST).
FST IV: Reverse J. In stands undergoing seed regeneration, samplings co-exist with the dominant canopy, so that the frequency of stems exponentially decreases towards the larger classes resulting on a reverse J-shaped histogram. Its perfect generalization into a negative exponential or a power function (Hett and Loucks, 1976) may be difficult to attain in practice, and usually many peaks are present if small bin sizes are considered, like in the present study. Usually this situation can be identified by a positively skewed histogram of diameters and a negatively skewed basal area distribution. This FST can also be subdivided, as parent trees coexist with saplings right after a disturbance opens a gap in the canopy, therefore producing a descending histogram with a small peak in the dominant canopy (FST $I V a)$. This later evolves into a more ideal reverse J-shaped histogram (FST IVb).

\subsection{Parametric family of diversity indices}

The complexity of forest structures was measured with a number of non-spatially explicit indices, i.e., those not accounting for tree positions but just relative frequencies of stems among diameter classes, calculated at plot-level. The first approach applied was to compute indices of diversity of size classes within a forest stand, considering that they could be feasible descriptors about the complexity of forest structure. In practice, this implies that indices which are traditionally used for describing species biodiversity, are adapted for their use to describe diversity, dominance or inequality among tree layers. The most straightforward descriptor of forest complexity would be to just state the number of size classes ( $s$ ) observed in the plot (richness). Other diversity indices describe the combined effect of both richness and relative abundances $\mathbf{p}=\left\{p_{i}\right\}$ of each size class $i$. When adapted to the study of tree size distributions, $\mathbf{p}$ can be defined as either the relative frequency of number of stems at each size class $\left(p_{i}=n_{i} / n\right.$, where $\left.\sum_{i=1}^{s} n_{i}=n\right)$, or their relative proportions of basal area $\left(p_{i}=g_{i} / g\right.$, where $\sum_{i=1}^{s} g_{i}=g$ ) per hectare. The abundance vector can also be computed from proportions of wood volume and above-groundbiomass, though in the present study they provided similar results as basal areas and led to the same conclusions, and have consequently been omitted.

Diversity indices are based on the probability that two randomly encountered trees belong to the same size class. The probability of double-encounter averages $1 / s$ if only considering the number of size classes present in that forest. It can also be argued that the probability is greater for the most abundant classes, and accordingly use the relative abundances of each size class $\left(p_{i}\right)$ as sample descriptors of this probability. When $\mathbf{p}$ is computed from basal area proportions, it is also considered that bigger trees will be more likely encountered. An averaging of this property is therefore given by Simpson's concentration index $\left(D_{S i}\right)$ :

$D_{S i}=1 / \sum_{i=1}^{S} p_{i}^{2}$

The diversity of tree sizes in a forest plot would increase if the uncertainty is higher, i.e., relative abundances are smaller or number of size classes is bigger. Consequently a reciprocal is calculated so that the index increases as diversity does. $D_{S i}$ is a good measure of dominance, since it would provide exceptionally low values if a single size class account for most abundance. Moreover, Shannon's diversity index $\left(H^{\prime}\right)$ considers not just the relative dominance of each class but also its rarity, measuring the uncertainty as weighted average surprisal $\left(-\operatorname{In} p_{i}\right)$ :

$H^{\prime}=-\sum_{i=1}^{s} p_{i} \operatorname{In}\left(p_{i}\right)$ 
On the other hand, it may be preferred to place even more importance on the dominance, and consider only the abundance of the most dominant class, neglecting the frequency of the most rare size classes. Following the same logic as in $D_{s i}$, the reciprocal of probability of the most abundant class is calculated in the Berger-Parker index $\left(D_{B P}\right)$ :

$D_{B P}=1 / \max \left(p_{i}\right)$

The choice of index usually depends on the properties to be described since, although the values of $H^{\prime}$ and $D_{S i}$ are affected by both richness and relative abundance, $H^{\prime}$ is more influenced by richness, whereas $D_{S i}$ is more biased towards dominance of the most abundant class (Lambshead et al., 1983). Diversity indices are therefore intermediate degrees in between measuring only richness with $s$, or just dominance in $D_{B P}$. These four indices described so far, $s, H^{\prime}, D_{S i}$, and $D_{B P}$, are closely inter-related, as they are specific cases of the generalized entropy of order $\alpha$ :

$H_{\alpha}=\frac{1}{1-\alpha} \operatorname{In} \sum_{i=1}^{s} p_{i}^{\alpha}$

Which can also be described by its corresponding diversity number (Hill, 1973):

$N_{\alpha}=e^{H_{\alpha}}$

As the entropy of order $\alpha=0$ is the number of size classes $s=N_{0}=e^{H_{0}}$; the entropy of order $\alpha=1$ equals to Shannon's $H^{\prime}=\operatorname{In}\left(N_{1}\right)=H_{1}$; and the entropy of order $\alpha=2$ is described in Simpson's index $D_{S i}=N_{2}=e^{-H_{2}}$. Note that we also may affirm that $D_{B P}=N_{\infty}$, as $\alpha=\infty$ would emphasize the proportion of the dominant class and $\alpha=-\infty$ would just describe how rare is the least abundant class (dominance versus rarity).

A diversity profile of a forest plot is therefore obtained by examining the continuum of either $H_{\alpha}$ or $N_{\alpha}$ along an increasing $\alpha$. Instead of calculating single indices directly, prior observation of a diversity profile showing the entire family of indices provides an insight into whether the generalized method used for comparing the forest plots is consistently describing the targeted concept of forest structure. In other words, a forest plot would have more diversity of size classes than another if its diversity values were greater throughout its profile, whereas in the case of intersecting profiles these plots would be non-comparable in terms of their diversity. There is also a variety of other methods for generalizing and ordering indices based on information theory, though we chose to test these only, as Liu et al. (2007) have demonstrated their equivalence.

\subsection{Intrinsic diversity ordering}

Another way of testing if two forest plots are comparable is to check whether contrasting their distributions is compatible with the definition of diversity itself. According to Patil and Taillie (1982), an initial situation in the structure of a forest plot (F1) can develop into an intrinsically more diverse state (F2) by a finite sequence of (a) introducing new size classes, (b) transferring abundances between two classes to make them more identical, or (c) it should remain equally diverse if just permuting the components of the abundance vector. When comparing two plots, this can be proven true if their accumulated dominance at each size class is always lower, i.e., their intrinsic diversity ordering is consistent. This is called the majorization method $I_{k}\left(k, M_{k}\right)$ (Solomon, 1979), and it is just one technique of many available for intrinsic diversity ordering. Liu et al. (2007) demonstrated that intrinsic diversity ordering methods are the most reliable procedure for ensuring that two forest plots are comparable. Arranging the components of $\mathbf{p}$ in descending order gives the ranked abundance vector $\mathbf{p}^{\#}=\left\{p_{k}^{\#}\right\}$ ), where $k$ is the rank of each size class, and hence $k=1, \ldots, s$ and $p_{1}^{\#}>p_{2}^{\#}>\cdots>p_{s}^{\#}$. A cumulative ranked abundance curve can be obtained:

$M_{k}=\sum_{j=1}^{k} p_{j}^{\#}(k=1, \ldots, s)$.

When F1 has intrinsically more diversity of size classes than F2, its intrinsic diversity profile $\left(I_{k}\right)$ lies completely below F2's. This complies with the definition of diversity as F2 may become F1 by either (a), (b) or (c). However, if their profiles intersect, there would be no intrinsic ranking between them. The diversity indices described above $\left(s, H^{\prime}, D_{S i}\right.$, and $\left.D_{B P}\right)$ are all based on this definition, becoming less weighted towards (a) for the entropy measures of higher the order. For this reason, if no intrinsic ordering is assured, these indices may be inconsistent in their measurement of diversity. It is noteworthy to mention that Swindel et al. (1987), Gove et al. (1995), and Solomon and Gove (1999) properly followed the theory of intrinsic diversity ordering when applying tail-sums for selecting among treatment alternatives, though there have been no recent examples of this approach being applied in the recent forestry literature.

\subsection{Intrinsic equitability ordering}

As explained, a measurement of diversity comprises both richness of size classes and the equitability of abundances among them (Studeny et al., 2011). There may be interest in studying the inequality among size classes independently from how many they are, i.e., the shape of the abundance vector. This can be done if richness is also rescaled into cumulative proportions, being $\mathbf{x}=\left\{x_{k}\right\}$, where $x_{k}=k / s$. Then, we can obtain the curve $P_{k}\left(x_{k}, M_{k}\right)$, which is also called the Lorenz curve, but with the difference that $\mathbf{p}^{\#}$ is more commonly arranged in ascending order instead (in this article, Lorenz curves are ranked in descending order to allow direct comparison with intrinsic diversity ordering curves). In fact, provided that all the forest plots had the same number of size classes, the Lorenz equitability ordering would be equivalent to the mentioned intrinsic diversity ordering methods (Lambshead et al., 1983). Conversely, as the intrinsic diversity ordering scale is normalized by richness, and ranks are arranged by cumulative proportions of total richness at plot level, $P_{k}$ becomes an intrinsic equitability ordering method.

\subsection{Parametric family of equitability indices}

Once the intrinsic equitability ordering of a plot has been constructed, we may suggest the possibility of also generalizing the method and drawing an equitability profile (Studeny et al., 2011). The entropy indices described above, $H^{\prime}$ and $D_{S i}$, also have their equitability counterparts, so-called Shannon's $\left(J^{\prime}\right)$ and Simpson's $\left(E_{S i}\right)$, evenness (Magurran, 2004). In order to also express equitability in the same terms as we did with the generalized entropy $H_{\alpha}$ and diversity number $N_{\alpha}$, we can observe that the traditional way of measuring $J^{\prime}$ equals:

$J^{\prime}=H^{\prime} / \operatorname{In}(s)=H_{1} / \operatorname{In}(s)=e^{H_{1}} / s=N_{1} / s=N_{1} / N_{0}$

Likewise $\left(E_{S i}\right)$, is:

$E_{S i}=D_{S i} / s=N_{2} / s=N_{2} / N_{0}$

This intuitively leads us to expressing generalized equitability numbers as (Hill, 1973):

$E_{\alpha, 0}=N_{\alpha} / N_{0}$

An equitability profile of a forest plot can therefore be observed in a similar manner as the diversity profile, by comparing the values of $E_{\alpha, 0}$ along an increasing $\alpha$. The intention would be 
to observe whether $J^{\prime}$ and $E_{S i}$ order forest structural types consistently. If, on the contrary, the empirical equitability profiles of forest plots intersect, $J^{\prime}$ and $E_{S i}$ would order structural types inconsistently.

\subsection{Individual trees instead of size classes}

The above-explained adaptation of the Lorenz curve to the discrete case can be useful for a study involving equitability among species (Wittebolle et al., 2009), which is essentially a categorical variable. It is also interesting to note how the Lorenz ordering fundamentally compares to the intrinsic diversity ordering methods, and thus it can also be called an intrinsic equitability ordering method (Studeny et al., 2011). However, it is a more common procedure to accumulate a continuous variable when computing Lorenz curves, and this is the case of the basal area $\mathbf{g}=\left\{g_{t}\right\}$, which is available for each individual tree within the plot. Hence, separating into discrete size classes is unnecessary, if the trees can themselves be ranked according to the variable used. In this approach, the concept of richness is no longer considered as the total number of size classes $s$, thus changing into the total number $n$ of trees in the plot. Likewise, instead of size class ranks $k$, we obtain tree size ranks $r$. Individual trees can be ranked according to their size in descending order $g^{\#}=\left\{g_{r}^{\#}\right\}$, where $r=1, \ldots, n$ and $g_{1}^{\#}>g_{2}^{\#}>\cdots>g_{n}^{\#}$, and these basal areas can be accumulated as:

$M_{r}=\sum_{j=1}^{r} p_{j}^{\#}(r=1, \ldots, n)$, where $p_{j}^{\#}=g_{j}^{\#} / g$

The Lorenz curve can be plotted to observe the relative increase in basal area accounted by each tree $P_{r}\left(x_{r}, M_{r}\right)$, being $x_{r}=r / n$ (as compared to $x_{k}=k / s$ for $P_{k}$ ). It is worth noting the analogy with $M_{r}$, and also express $x_{r}$ as:

$x_{r}=\sum_{j=1}^{r} p_{j}^{\#}(r=1, \ldots, n)$, where $p_{j}^{\#}=n_{j}^{\#} / n$

Eqs. (10) and (11) clearly show how $P_{r}$ is a normalization of the accumulated abundance in terms of basal area (can also be volume or biomass) expressed by the accumulated abundance in terms of number of trees, therefore observing the equitability of the measured variable ( $g$ and therefore $d b h$ ) independently from the total number of trees. As was the case for diversity indices, the use of single indices based on equitability ordering also requires a detailed analysis of Lorenz curves (Studeny et al., 2011; Weiner and Solbrig, 1984).

\section{Results}

Figs. 2 and 3 illustrate the diversity and equitability profiles, respectively. Fig. 4 shows the Lorenz curves computed for individual trees. In Figs. 2 and 3, a and b refer to profiles computed from a vector of abundances calculated as relative frequencies of stems, whereas $\mathrm{c}$ and $\mathrm{d}$ refer to abundances expressed as proportions of basal area. The same plots as depicted in Fig. 1 were used to construct all the diversity and equitability profiles presented in this article, allowing direct comparison of all figures.

\subsection{Diversity profiles}

Fig. 2a and c show the intrinsic diversity ordering $I_{k}\left(k, M_{k}\right)$ of the FSTs considered, and make it possible to observe if their abundance distribution is consistent with the definition of diversity. Under the diversity scale given by majorization (see Section 2.3), a forest plot has more diversity of size classes than another if its curve lies completely below. For instance, results showed that FST IVb has intrinsically more diversity of size classes than FST IVa, whether abundances are considered from stem frequencies or basal areas. However, many of these profiles intersected, indicating for example that FST III and FST IVa are intrinsically non-comparable using this diversity scale.

Fig. $2 \mathrm{~b}$ and $\mathrm{d}$ are the diversity numbers obtained by these same field plots, and therefore they illustrate if diversity indices order them logically and consistently. The gray boxes in the background of these figures illustrate the correspondence between the order of entropy $(\alpha)$ and the diversity indices described, which are explicitly indicated at the bottom. Profiles of more diverse plots lie above less diverse ones, demonstrating that a higher value is obtained by each specific diversity index. Using the same examples as before, it can be observed that FST IVb consistently obtained higher values than FST IVa for all the diversity indices of the parametric family. The opposite situation was found when comparing FST III and FST $I V a$, since $s$ and $D_{S i}(\alpha=0$ and 2, respectively) evaluated their relative diversity of size classes differently, as a consequence of their lack of intrinsic ordering.

In general, profiles were comparable when they were originated from similarly-shaped distributions. Results suggested that exponentially decreasing stem distributions, namely bimodal and reverse J (FSTs III and IV), were usually comparable. It is also possible to inter-compare among profiles originated from distributions that can be approximated to a normal distribution (FSTS I). On the other hand, profiles from even-sized plots intersected those from reverse J-shaped ones, indicating that they are non-comparable. Likewise, when computing abundance from basal areas, negatively skewed distributions (FSTs II and IV) were non-comparable to the normal and bimodal ones (FSTS I and III). For this reason, $H^{\prime}$ and $D_{S i}$ seemed meaningless when comparing all plots at once. Otherwise, diversity indices were a suitable method for comparing plots within a same FST. Overall, the intersecting curves demonstrated that $s, H^{\prime}, D_{S i}$, and $D_{B P}$, categorized the plots by their structural diversity inconsistently, and they cannot be relied upon to provide a logical order of the empirical plots according to their structural complexity.

\subsection{Equitability profiles}

Fig. 3a and c show the intrinsic equitability ordering $P_{k}\left(x_{k}, M_{k}\right)$ of the plots, i.e., their Lorenz ordering by size classes. The gray diagonal line represents the ideal situation in which abundances are equal among all the size classes present, and therefore curves closer to the diagonal signify more equitability among size classes. Profiling equitability resulted in fewer intersections than diversity, and curves were more distant to each other. However, it should be noted that their intrinsic equitability ordering was not prone to intersections either, as FST III and FST IVb lacked intrinsic equitability ordering when abundances were considered from stem frequencies. In general, the concept of equitability suggested that plots belonging to FST I had more inequality among their size classes than those within FST IV.

Fig. $3 b$ and $d$ are the equitability numbers obtained by these plots, and therefore they illustrate if equitability indices, i.e., diversity numbers normalized by richness, order them logically and consistently. The correspondence with the equitability indices considered is also shown by the gray boxes in the background of these figures; therefore, the curves at the top signify higher values of these indices. Most results were consistent, and only small differences between $J^{\prime}$ and $E_{S i}$, ( $\alpha=1$ and 2, respectively) were obtained for those plots lacking intrinsic ordering. Equitability in stem frequencies clearly discriminated even-sized and uniform plots (FST $I$ and II) from bimodal and reverse J (FST III and IV). When basal areas are used the separation is less clear, but the order remained similar except in the case of uniform (FST II) and bimodal (FST III) 

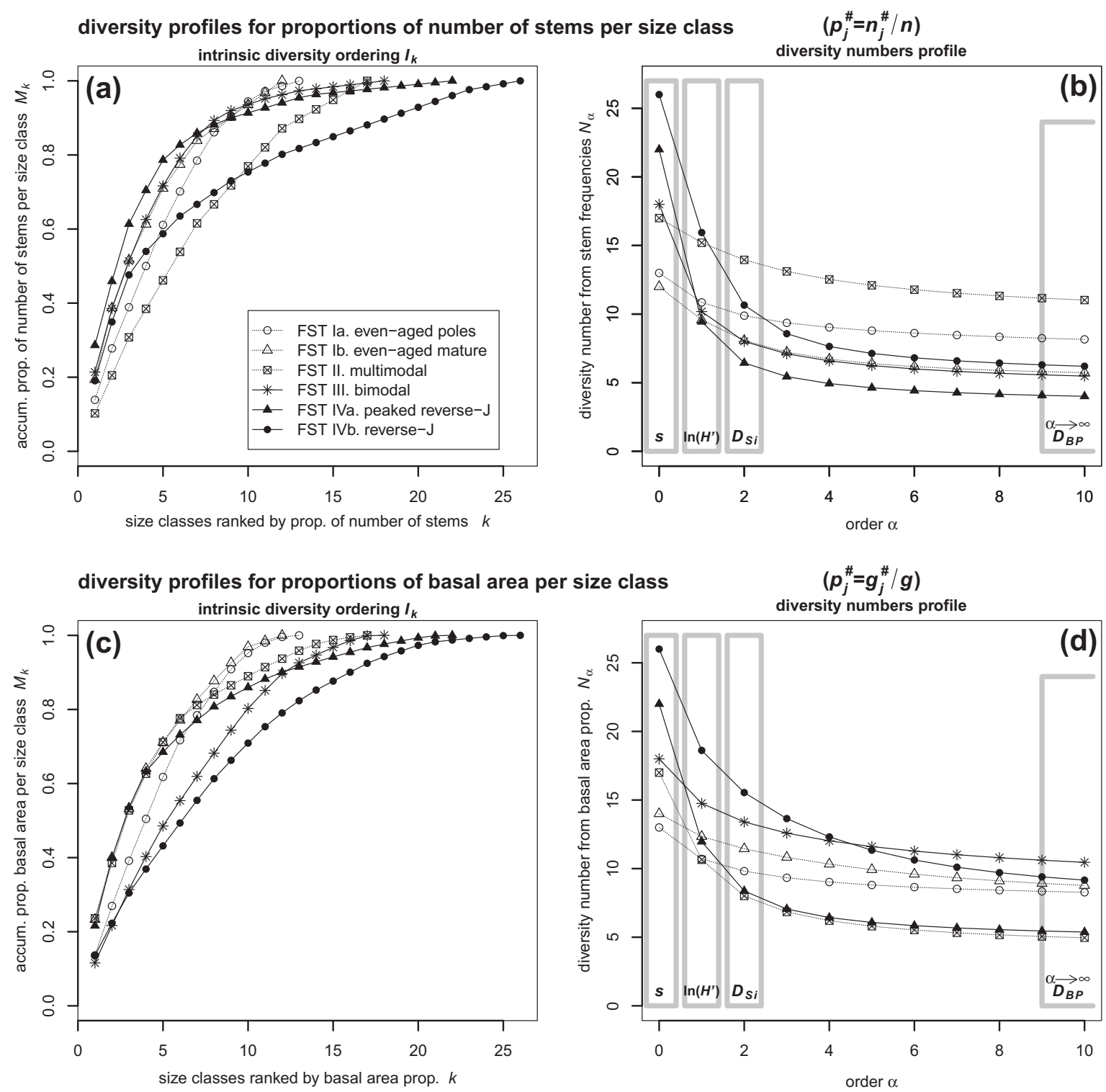

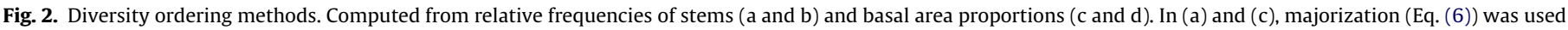

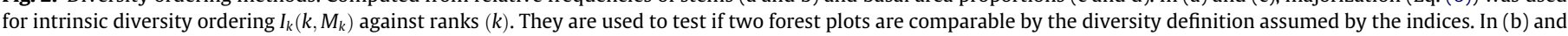

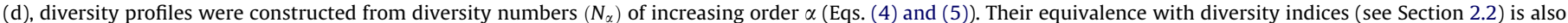

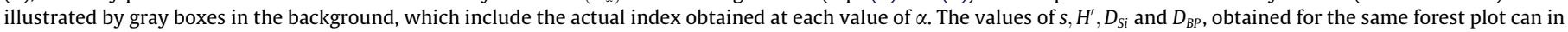
this way be evaluated simultaneously along its diversity profile.

structures, which seem to be evaluated very differently depending on how abundances are computed.

\subsection{Lorenz curves from individual tree sizes}

Fig. 4 shows the Lorenz curves obtained when trees are ranked individually $P_{r}\left(x_{r}, M_{r}\right)$. In order to clarify the interpretation of this figure, the Lorenz curves of theoretical distributions were included in gray color. Like in Fig. 3a and c, the diagonal is the line-of-absolute-equality (gray dotted line), although in this case it is obtained by any forest stand with all trees of equal size, regardless of whether they are big or small. The opposite situation would be a maximally bimodal distribution (gray dashed line), characterized by a theoretical forest plot with two cohorts of equal basal areas, so that few large trees account for half the basal area in the dominant canopy and the other half is cumulated from lots of small saplings. In the particular case of applying Lorenz curves to the study of tree size distributions, it is of particular interest to observe as well the ideal situation represented by the line-of-perfect-uniformity (gray solid line), obtained from a theoretical sequence of trees with steady increase in their diameters along the whole range.

The best results were obtained when the intrinsic equitability ordering was observed for the individual trees (Fig. 4), instead from size classes. Lorenz curves generated from individual trees clearly showed the best separability among FSTs. Also, the forest plots were shown in an order which seemed more logical according to their structural properties.

\section{Discussion}

\subsection{Applicability of methods based on diversity}

The results reveal that diversity indices are not applicable for all studies of the complexity of forest structure. The field plots did not 

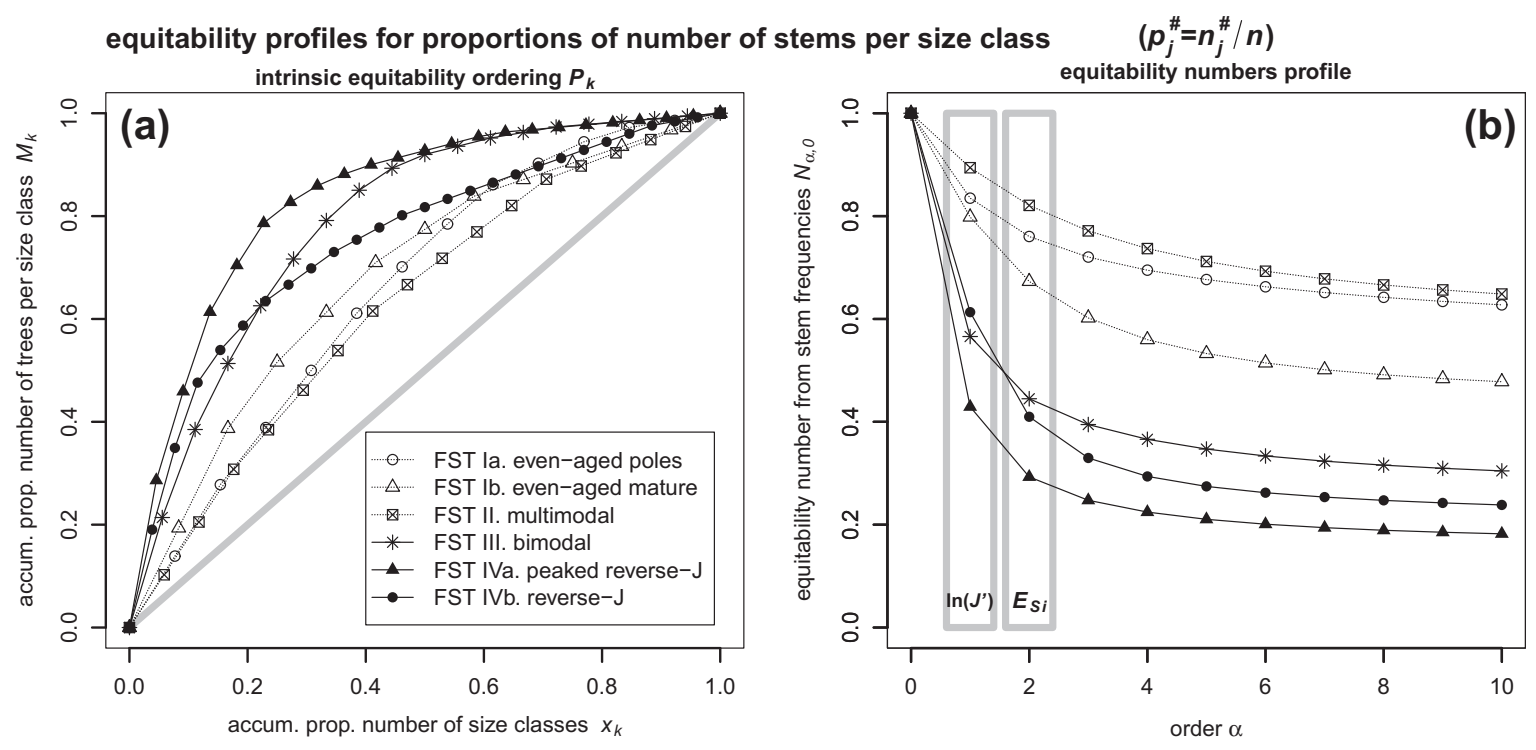

equitability profiles for proportions of basal area per size class intrinsic equitability ordering $P_{k}$
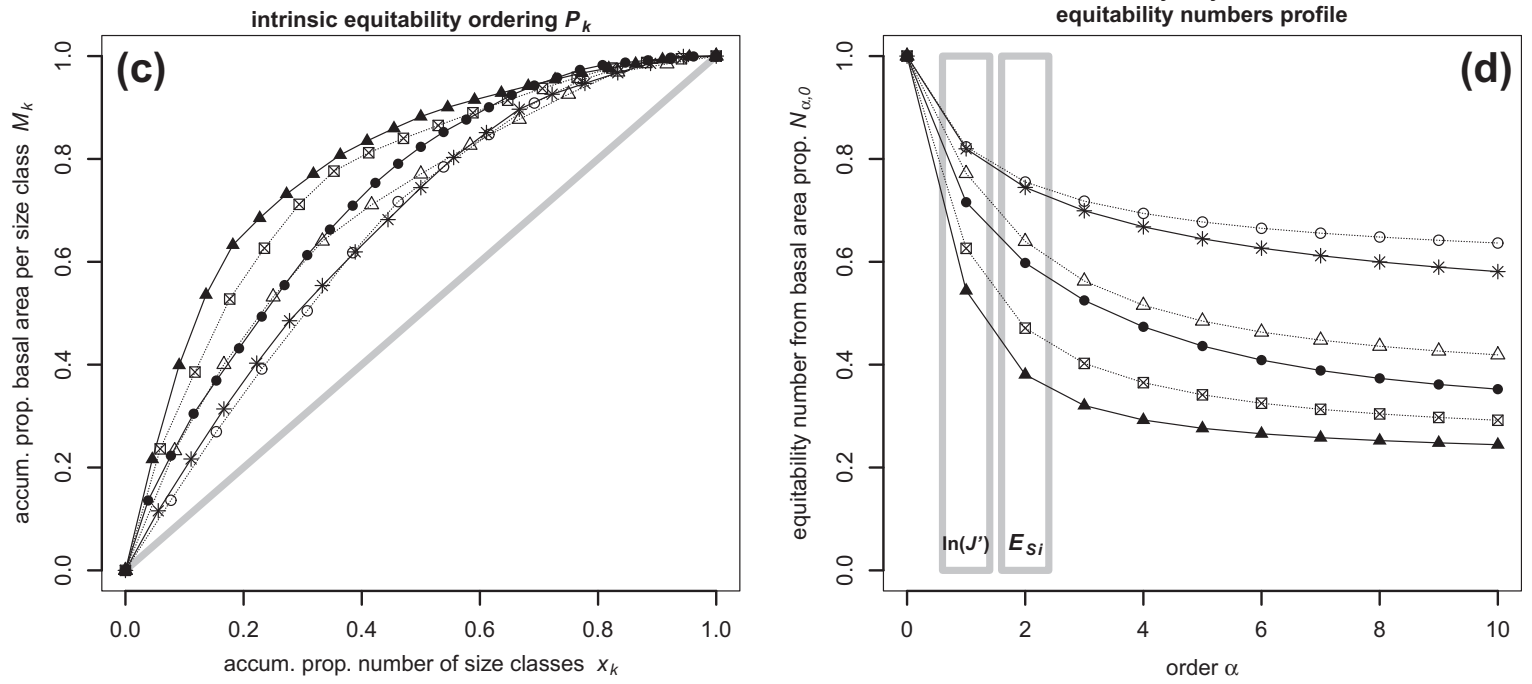

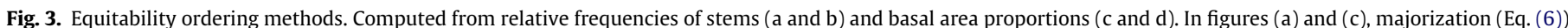

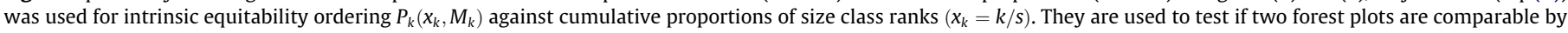

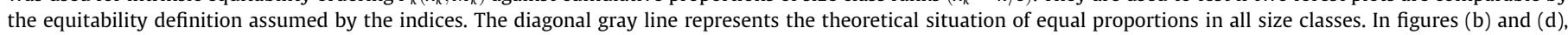

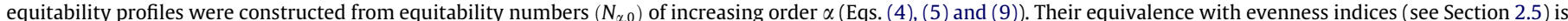

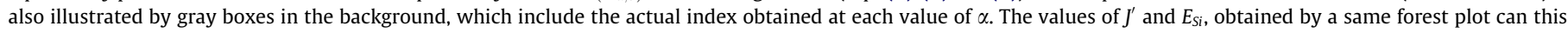
way be evaluated simultaneously along its equitability profile.

follow the same intrinsic diversity ordering (Fig. 2a and c), and therefore a concise indicator based on this assumption is inadequate for their inter-comparison. This conclusion would also apply to other diversity indices based on information theory, e.g., McIntosh or Brillouin (cf., Lambshead et al., 1983), as they are grounded on the same assumptions.

On the other hand, not all cases led to non-comparable diversity ordering. Diversity indices seem perfectly fine to be used for comparing among even-sized stands, or analyzing the success of regeneration in reverse J-shaped ones. For this reason, these results do not necessarily invalidate the conclusions reached by previous studies using Shannon, or other indices alike. For instance, forest plots partitioned into larger bin widths are more likely to be intrinsically comparable, as it could be the case of McArthur and McArthur (1961), who divided the canopy into just three vertical strata. It can also be feasible to contrast distributions of similar shape, like Buongiorno et al. (1994) and Sullivan et al. (2001), who compared among reverse J-shaped stands to test the effects of disturbances and management alternatives in uneven-sized stands. However, the absence of intrinsic diversity ordering may be the reason why it has been reported that Shannon index may lead the same value for very different FSTs (Harper et al., 2003; Kint et al., 2000; Lexerød and Eid, 2006; Rouvinen and Kuuluvainen, 2005). Using circular plots of variable radii (Sullivan et al., 2001 ) can be another source for confusion, as plot size influences the computation of indices affected by sample richness (Barbeito et al., 2009; James and Wamer, 1982).

O'hara et al. (2007) found that uneven-sized stands did not necessarily obtain better values of diversity indices than even-sized ones, but their conclusions should be taken cautiously as these indices have been demonstrated unreliable for such comparison. Diversity indices may be consistent for field plots in other studies, but Fig. 2 proves the need for accompanying any index with corresponding diversity profiles, as there is potential for inconsistencies. 


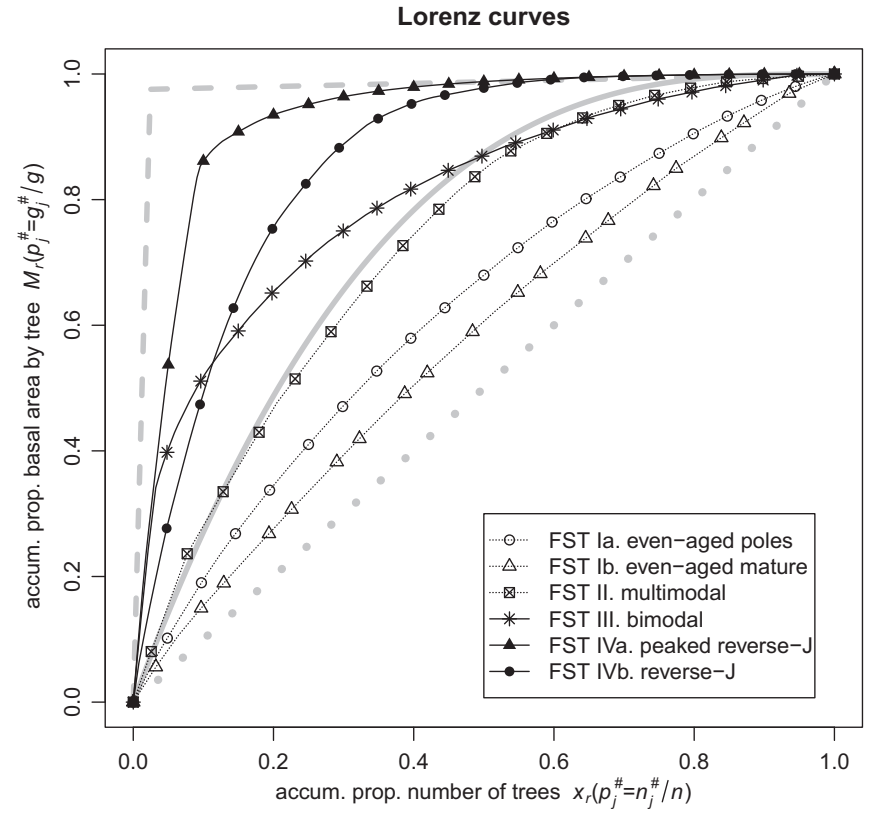

Fig. 4. Lorenz curves, i.e., intrinsic equitability ordering of individual trees by means of majorization $P_{r}\left(x_{r}, M_{r}\right)$. Accumulated abundances $\left(M_{r}\right)$ computed for ranked basal areas $\left(p_{r}^{\#}=g_{r}^{\#} / g\right)$ in Eq. (10) are plotted against the cumulative proportions of tree size ranks $\left(x_{r}=r / n\right)$ in Eq. (11). Gray lines signify theoretical distributions: line-of-absolute-equality (dotted), line-of-perfect-uniformity (solid), maximally bimodal (dashed)

The validity of results based in diversity among size classes cannot be confirmed until diversity profiles are evaluated and empirical data assured comparable. We suggest that future studies include the assessment of the intrinsic ordering of the data with diversity and equitability profiles, prior to reporting any results on single indicators and reaching any conclusions.

As an alternative to always plotting the intrinsic ordering when using a single index, an analytical approach could also be performed, by programmatically assuring that $M_{k}$ consistently increases/decreases for all $k$ (Gove et al., 1992), or testing for Shur concavity (Gattone and Battista, 2009). Gove et al. (1995) applied this method to assure diversity of basal area proportions among size classes, though using the right tail-sum method $R_{k}\left(k, T_{k}\right)$ instead of majorization. For each size class, they accumulated the proportion of abundance in the remaining $s-k$ least abundant size classes (Patil and Taillie, 1982):

$T_{k}=\sum_{j=k+1}^{s} p_{k}^{\#}, \quad$ for $k=1, \ldots, s-1$

When compared to Eq. (6), it becomes apparent how $T_{k}$ accentuates the relative abundance of the least common size classes (rarity), whereas $M_{k}$ emphasizes the importance of most common ones (dominance). Both methods lead to the same result (Liu et al., 2007) and they are only conceptually different, and therefore choosing either one is just a matter of whether diversity is used with the intention of describing dominance or rarity.

Future research on diversity profiling may consider some plotting alternatives which enhance visual identification of intersecting profiles. Lambshead et al. (1983) suggested the use of $k$-dominance curves $K_{k}\left(\operatorname{In} k, M_{k}\right)$ showing percentages of cumulated dominance, as the logarithmic scale straightens the curves and their separability or intersections becomes easier to assess. When the purpose is to compare management alternatives against a common baseline, another useful approach was developed by Swindel et al. (1987) as comparative diversity profiles $C_{k}\left(M_{k}, M_{k}^{\prime}\right)$. These profiles directly compare the intrinsic ordering of one plot $\left(M_{k}\right)$ against another $\left(M_{k}^{\prime}\right)$, and therefore serves as an intersection test in itself. The authors recommend these in future studies, though those used in this article allowed direct comparison between majorization and Lorenz ordering. From the statistical point of view, there are also some improvements that could be taken into account in further research. For instance, as cumulated dominance was computed from sample plots, the profiles themselves can be considered as their average estimate, with corresponding confidence intervals. For this reason, Gove et al. (1992) used a jack-knifing method to infer whether two profiles significantly intersect. The most important consequence is that the confidence range is variable along the profile, and therefore the significance of intersections depends upon where profiles cross. Future research may also investigate the effect of other assumptions underlying the use of relative abundances as descriptors of the probability for double-encounter in diversity indices. Sample estimators of richness and abundance are only asymptotically $(n \rightarrow \infty)$ unbiased (Sheldon, 1969), and there is a premise of no spatial autocorrelation which is implicitly contravened by tree competition. Sample plot size can be optimized to minimize these effects, and excessively fine scales should in general be avoided (Barbeito et al., 2009).

\subsection{Diversity vs. equitability}

The intrinsic equitability ordering (Fig. 3a and c) observed for the empirical plots suggests that equitability is a concept more suited for comparing them than diversity. As a consequence, diversity indices seemed less reliable for studying forest structures that their equitability counterparts. Contradictorily, Shannon's index is the most popular among all alternative indices for describing structural complexity of forests. Diversity indices give a higher or equal value from an initial situation by (a) incorporating more classes, (b) transferring abundances, or (c) relabeling classes (Patil and Taillie, 1982). As explained, the higher the order of an entropy measure is, the less weighted towards (a) it becomes. When using an equitability measure, the values depend only on (b) and (c), whereas the component of (a), which is expressed alone in the entropy of order zero, is removed. These definitions for the concepts of both diversity and equitability should be born in mind when using these indices, as they will not apply if the intrinsic orderings intersect. However, this does not mean that indices cannot be used in any case if intrinsic ordering is not assured. It may be the case that just practical criteria needs to be taken into account, such as their discriminant ability and capacity for ordering in a logical ranking (Lexerød and Eid, 2006; Staudhammer and LeMay, 2001).

\subsection{Number of stems vs. basal areas}

Either option for computing the abundance vector $\mathbf{p}$ showed different advantages in their intrinsic ordering, as number of stems resulted in fewer intersections (Fig. 3a) and Lorenz curves from basal areas were more separated to each other (Fig. 3c), providing more potential for FST discrimination. Solomon and Gove (1999) recommended using variables strongly influenced by larger trees, such as basal area volume or biomass, so that the resulting indices become more representative with regards to forest management. A criterion of practicability for the aim of each management practice should prevail over the mathematical properties of the distributions, though these should also be observed in accordance with the methods chosen. For example, both methods coincided in evaluating FST I plots as having more equitability among their size classes than FST IV ones, but they disagreed in the assessment of FSTS II and III. The choice of either stem density or basal area therefore depends on whether the forest manager wishes to emphasize the success of recruitment or the continuity of canopy strata. 
Results are also differently evaluated if number of stems or basal areas are considered. In all cases, curves closer to the diagonal result in lower GC and higher $J^{\prime}$ and $E_{S i}$. However, depending on the abundance vector used, a different forest structure is defined as the most equitable. Absolute equitability would be obtained by a theoretical forest plot with uniformly distributed diameters in Fig. 3a, whereas at Fig. $3 c$ the diagonal would instead represent exponentially decreasing stems frequencies showing uniformly distributed basal areas. This distribution was simulated by Lexerød and Eid (2006) suggesting that a reverse J would obtain higher values for equitability indices. In our field plots such distribution was not observed in practice, and basal areas were seemly more evenly segregated in a bimodal (FST III) than in a negative exponential (FST IVb) (Fig. 1). As a consequence, the highest values of $J^{\prime}$ and $E_{S i}$ were obtained by FSTS I and III (Fig. 3d).

Overall, after considering all the diversity and equitability profiles, we conclude that using the information from stem frequency and basal area simultaneously (Fig. 4) can be a better approach than one of them alone (Fig. 3a and c). This got signified by Eqs. (10) and (11), which exemplify how information on both the distribution of stems and their basal areas is contained within the axes in Fig. 4. By observing in Fig. 1 the distributions of stem frequency and basal area of the different FSTs considered, it can be intuitively presumed that neither of them alone contains enough information for reliably distinguishing among them. For instance, FST IV would not be discriminated from FST III by a descriptor of histogram's shape, while describing the skewness of the basal area distributions would also fail to differentiate it from FST II. The Lorenz curves in Fig. 4 combine information from both stem/basal area distributions, and therefore are more feasible for FST discrimination. Lorenz curves in Fig. 4 have to be interpreted differently, as there is a semantic difference between considering equality in tree sizes or evenness among size classes. Theoretical stands in Fig. 4 with uniform distribution (gray solid line) and all trees equal (gray dotted line), would both coincide with the diagonal at Fig. 3a. They are, however, very different from the silvicultural point of view, as they belong to FST II and I respectively.

\subsection{Interpretation of Lorenz curves from tree sizes}

In light of the results shown in Fig. 4, we suggest that a particular interpretation of Lorenz curves applies when they are computed with the purpose of analyzing the structural properties of the canopy. Lorenz curves computed from individual tree sizes should not only be compared to the traditional line-of-absoluteequality (gray dotted line), but also to the line-of-perfect-uniformity (gray solid line). This line can be used as a reference for studying forest dynamics and stand development. Note that GC equals 0.5 for an asymptotically uniform distribution. Hence, for forest plots obtaining $\mathrm{GC}>0.5$, starting from a peaked reverse $\mathrm{J}$ (FST IVa), the success of natural regeneration and ingrowth can be indicated by decreasing GCs. Also, the asymmetry coefficient would shift from $S<1$ to $S>1$ (for concave Lorenz curves, but from $\mathrm{S}>1$ to $\mathrm{S}<1$ for convex ones) as an indication of achieved recruitment (FST III) from a reverse J (FST IVb). On the other hand, GC $<0.5$ denotes even-sized forest structures (FST I), and therefore they can be used to study self-thinning processes taking place when stand development is determined by competition for light and space (Montgomery and Chazdon, 2001). Conversely, disturbances would be indicated by a symmetric shift of the Lorenz curve to either side the line-of-perfect-uniformity, as for example a gap opened in the canopy would lead from FST Ib to FST IVa. This would indicate any type of disturbance increasing mortality rate for larger trees (Coomes et al., 2003), whether naturally (e.g., windthrow) or artificially (e.g., thinning from above). Then, although FST Ib and FST IVa are on opposite areas of the plot, we can consider them to be quite similar in terms of the dominance of the upper canopy layer.

\subsection{Selecting indicators of structural complexity of forests}

Reasoning from the results presented in this article, any indicators based on Lorenz curves can be well suited for describing the complexity of forest structures, such as GC and $\mathrm{CH}$. Values of GC should be interpreted according to their theoreticals, as GC $=0$, 0.5 and 1 for the diagonal, uniform and maximally bimodal, respectively. This property is invariant of the species considered and applicable to other forest ecosystems, as is consistent with recent research by Duduman (2011) and Klopcic and Boncina (2011) which also ordered FSTs by increasing GCs as: even-sized, uniform, bimodal and reverse J. As Lorenz curves may cross, it is advisable to accompany GC with the Lorenz asymmetry coefficient S (Damgaard and Weiner, 2000). Furthermore, when developing his $\mathrm{CH}$, De Camino (1976) also paid special attention to this line-of-perfect-uniformity. $\mathrm{CH}$ equals 1 for a forest stand belonging to this line, and higher values are given as the distance between this line and the Lorenz curve increases in either direction. This index was initially conceived for ranking proportions of volume, though there is no theoretical limitation in using basal areas instead.

Using diversity indices is discouraged, though they can still be valid if it can be assured that all comparisons are performed at either side of the line-of-perfect-uniformity, as the two groups were demonstrated to be non-comparable by the definition of diversity. Apart from GC, a rapid analytical method for assuring this restriction can be to compare sample variance in diameters against the variance of a uniform density function. Lorenz curves under this line would have smaller variances than a theoretical uniform, whereas those over this line would be higher. This property has an important analogy with the rationale used by Staudhammer and LeMay (2001) in their structure index based on variance (STVI). For this reason, STVI is also recommended as an index coherent with the properties stated in this article, as it also describes the distance from this line-of-perfect-uniformity.

Equitability of basal areas among size classes, measured by means of Simpson's evenness index, is also a criterion with good potential for FST discrimination (Fig. 3d). In light of our results, $E_{S i}$ is probably the best of the alternatives considered in this article, though still difficult to interpret. Although equitability was considered as the subtraction of the richness component from diversity, it should nevertheless be noticed that equitability indices are not completely prone to a dependency on $s$ (Sheldon, 1969). Lexerød and Eid (2006) also found that the problem of using Margalef's index was its dependency on $n$, which prevents stands of differing densities to be comparable. These are more arguments in favor of using individual trees instead of size classes, as sample size increases and stands become density-normalized.

\section{Conclusions}

The concept of diversity is inadequate for describing the structural complexity of forests, and therefore adapting species diversity indices to measure entropy among tree size classes is incorrect. Intersecting diversity profiles from various forest plots demonstrated that they are non-comparable by the definition of diversity. Hence, this practice should be avoided in future studies, or otherwise intrinsic ordering must be properly assured and reported. Evaluating equitability was demonstrated more satisfactory than diversity for this purpose, and there is no conceptual mistake in describing structural complexity of forests with a measurement on the evenness among its size classes. Nonetheless, results were more satisfactory when equitability was evaluated 
from individual trees instead of size classes in the Lorenz curve, as information of the both distribution of stems and their basal areas was simultaneously included. However, the Lorenz curves were not totally prone to intersections either, and therefore it is advised to observe always the curves rather than using a simple index. Whenever a simple indicator is required, GC (accompanied by S), $\mathrm{CH}$ and STVI are recommended, as they are coherent with the intrinsic equitability ordering of the empirical data. Further efforts should focus on investigating the applicability of these recommended indices for more accurate estimation in forest growth modeling, or for choosing among management alternatives. It may also be worth using Lorenz curves for investigating the different dynamics of various forest ecosystems.

\section{Acknowledgements}

Rubén Valbuena's work is funded by Metsähallitus (Finnish Forestry Commission) Grant and the Foundation for European Forest Research (FEFR). Authors wish to thank Dr. Gove (USDA Forest Service) for his useful comments over the previous version of the article. Tim Green (European Forest Institute) and an anonymous reviewer carefully proofread and improved the style of this article. The members of the Research Group for Sustainable Forest Management (Technical University of Madrid) are also thanked for their collaboration in the field campaign. This study was also supported by strategic funding from the University of Eastern Finland.

\section{References}

Bachofen, H., Zingg, A., 2001. Effectiveness of structure improvement thinning on stand structure in subalpine Norway spruce (Picea abies (L.) Karst.) stands. For. Ecol. Manage. 145, 137-149.

Barbeito, I., Cañellas, I., Montes, F., 2009. Evaluating the behavior of vertical structure indices in Scots pine forests. Ann. For. Sci. 66, 710p1-710p10.

Buongiorno, J., Dahir, S., Lu, H., Lin, C., 1994. Tree size diversity and economic returns in uneven-aged forest stands. For. Sci. 40, 83-103.

Cienciala, E., Korhonen, K., 2011. Indicator 1.3 Age structure and/or diameter distribution of forest. In: FOREST EUROPE, UNECE, FAO (Eds.), State of Europe's forests 2011: status and trends in sustainable forest management in Europe. FOREST EUROPE Liaison Unit. Ås, Norway, pp. 24-25.

Coomes, D.A., Duncan, R.P., Allen, R.B., Truscott, J., 2003. Disturbances prevent stem size-density distributions in natural forests from following scaling relationships. Ecol. Lett. 6, 980-989.

Damgaard, C., Weiner, J., 2000. Describing inequality in plant size or fecundity. Ecology 81, 1139-1142.

De Camino, R., 1976. Determinación de la homogeneidad de rodales. Bosque 1, 110 115.

Duduman, G., 2011. A forest management planning tool to create highly diverse uneven-aged stands. Forestry 84, 301-314.

Gattone, S.A., Battista, T.D., 2009. A functional approach to diversity profiles. J. Roy. Stat. Soc. Ser. C (Appl. Stat.) 58, 267-284.

Gove, J.H., Martin, C.W., Patil, G.P., Solomon, D.S., Hornbeck, J.W., 1992. Plant species diversity on even-aged harvests at the Hubbard Brook Experimental Forest: 10 year results. Can. J. For. Res. 22 (11), 1800-1806.

Gove, J.H., Patil, G.P., Taillie, C., 1995. A mathematical programming model for maintaining structural diversity in uneven-aged forest stands with implications to other formulations. Ecol. Model. 79, 11-19.

Harper, K., Boudreault, C., DeGrandpré, L., Drapeau, P., Gauthier, S., Bergeron, Y., 2003. Structure, composition, and diversity of old-growth black spruce boreal forest of the Clay Belt region in Quebec and Ontario. Env. Rev. 11, S79-S98.

Hett, J.M., Loucks, O.L., 1976. Age structure models of Balsam Fir and Eastern Hemlock. J. Ecol. 64, 1029-1044.

Hill, M.O., 1973. Diversity and evenness: a unifying notation and its consequences. Ecology 54, 427-432.

Hurlbert, S.H., 1971. The nonconcept of species diversity: a critique and alternative parameters. Ecology 52, 577-586.
James, F.C., Wamer, N.O., 1982. Relationships between temperate forest bird communities and vegetation structure. Ecology 63, 159-171.

Kint, V., Lust, N., Ferris, R., Olsthoorn, A.F.M., 2000. Quantification of forest stand structure applied to Scots Pine (Pinus sylvestris L.) forests. Invest. Agr. Sist. Recur. For. Fuera de Serie 1, 147-163.

Klopcic, M., Boncina, A., 2011. Stand dynamics of silver fir (Abies alba Mill.)European beech (Fagus sylvatica L.) forests during the past century: a decline of silver fir? Forestry 84, 259-271.

Knox, R.G., Peet, R.K., Christensen, N.L., 1989. Population dynamics in Loblolly Pine stands: changes in skewness and size inequality. Ecology 70, 1153-1167.

Lähde, E., Laiho, O., Norokorpi, Y., Saksa, T., 1991. The structure of advanced virgin forests in Finland. Scand. J. For. Res. 6, 527-537.

Lambshead, P.J.D., Platt, H.M., Shaw, K.M., 1983. The detection of differences among assemblages of marine benthic species based on an assessment of dominance and diversity. J. Nat. Hist. 17, 859-874.

Lei, X., Wang, W., Peng, C., 2009. Relationships between stand growth and structural diversity in spruce-dominated forests in New Brunswick, Canada. Can. J. For. Res. 39, 1835-1847.

Lexerød, N.L., Eid, T., 2006. An evaluation of different diameter diversity indices based on criteria related to forest management planning. For. Ecol. Manage. 222, 17-28

Liu, C., Whittaker, R.J., Ma, K., Malcolm, J.R., 2007. Unifying and distinguishing diversity ordering methods for comparing communities. Popul. Ecol. 49, 89100

Magurran, A.E., 2004. Measuring Biological Diversity. Blackwell Science, Oxford, UK. McArthur, R.H., McArthur, J.W., 1961. On bird species diversity. Ecology 42, 594598

Mc Elhinny, C., Gibbons, P., Brack, C., Bauhus, J., 2005. Forest and woodland stand structural complexity: its definition and measurement. For. Ecol. Manage. 218, $1-24$

McRoberts, R.E., Winter, S., Chirici, G., Hauk, E., Pelz, D.R., Moser, W.K., Hatfield, M.A., 2008. Large-scale spatial patterns of forest structural diversity. Can. J. For. Res. 38, 429-438.

Montgomery, R.A., Chazdon, R.L., 2001. Forest structure, canopy architecture, and light transmittance in tropical wet forests. Ecology 82, 2707-2718.

Motz, K., Sterba, H., Pommerening, A., 2010. Sampling measures of tree diversity. For. Ecol. Manage. 260, 1985-1996.

Neumann, M., Starlinger, F., 2001. The significance of different indices for stand structure and diversity in forests. For. Ecol. Manage. 145, 91-106.

O'hara, K.L., Hasenauer, H., Kindermann, G., 2007. Sustainability in multi-aged stands: an analysis of long-term plenter systems. Forestry $80,163-181$

Patil, G.P., Taillie, C., 1982. Diversity as a concept and its measurement. J. Am. Stat. Assoc. 77, 548-561.

Pommerening, A., 2002. Approaches to quantifying forest structures. Forestry 75 305-324.

Rouvinen, S., Kuuluvainen, T., 2005. Tree diameter distributions in natural and managed old Pinus sylvestris-dominated forests. For. Ecol. Manage. 208, 45-61.

Sheldon, A.L., 1969. Equitability indices: dependence on the species count. Ecology 50, 466-467.

Solomon, D.L., 1979. A comparative approach to species diversity. In: Grassle, J.F., Patil, G.P., Smith, W., Taillie, C. (Eds.), Ecological Diversity in Theory and Practice. International Cooperative Publishing House, Fairland, Maryland, USA, pp. 29-35.

Solomon, D.S., Gove, J.H., 1999. Effects of uneven-age management intensity on structural diversity in two major forest types in New England. For. Ecol. Manage. 114, 265-274.

Staudhammer, C.L., LeMay, V.M., 2001. Introduction and evaluation of possible indices of stand structural diversity. Can. J. For. Res. 31, 1105-1115.

Sterba, H., Ledermann, T., 2006. Inventory and modelling for forests in transition from even-aged to uneven-aged management. For. Ecol. Manage. 224, 278-285.

Studeny, A.C., Buckland, S.T., Illian, J.B., Johnston, A., Magurran, A.E., 2011. Goodness-of-fit measures of evenness: a new tool for exploring changes in community structure. Ecosphere 2 (Article 15).

Sullivan, T.P., Sullivan, D.S., Lindgren, P.M.F., 2001. Stand structure and small mammals in young lodgepole pine forest: 10-year results after thinning. Ecol. Appl. 11, 1151-1173.

Swindel, B.F., Conde, L.F., Smith, J.E., 1987. Index-free diversity orderings: concept, measurement, and observed response to clearcutting and site-preparation. For. Ecol. Manage. 20, 195-208.

Weiner, J., Solbrig, O., 1984. The meaning and measurement of size hierarchies in plant-populations. Oecologia 61, 334-336.

Wittebolle, L., Marzorati, M., Clement, L., Balloi, A., Daffonchio, D., Heylen, K., De Vos, P., Verstraete, W., Boon, N., 2009. Initial community evenness favours functionality under selective stress. Nature 458, 623-626.

Zenner, E.K., 2005. Development of tree size distributions in Douglas-Fir forests under differing disturbance regimes. Ecol. Appl. 15, 701-714. 